\title{
No Correlation Between Serum Markers and Early Functional Outcome After Contemporary THA
}

\author{
Kirsten L. Poehling-Monaghan MD, Michael J. Taunton MD, Atul F. Kamath MD, \\ Robert T. Trousdale MD, Rafael J. Sierra MD, Mark W. Pagnano MD
}

Published online: 27 May 2016

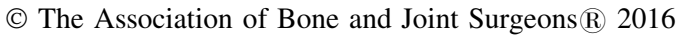

\begin{abstract}
Background Serum markers of inflammation and muscle damage have shown clinical utility in some areas of medicine, but their value in determining the invasiveness or in predicting the early functional outcomes after total hip arthroplasty (THA) has not been demonstrated.
\end{abstract}

\footnotetext{
One of the authors (MWP) or a member of his immediate family, has or may receive payments or benefits, during the study period, an amount of USD 100,001 to USD 1,000,000 from Stryker Orthopedics (Mahwah, NJ, USA). One of the authors (RTT) or a member of his immediate family, has or may receive payments or benefits, during the study period, an amount of USD 10,000 to USD 100,000 from DePuy Orthopedics (Warsaw, IN, USA). One of the authors (RJS) or a member of his immediate family, has or may receive payments or benefits, during the study period, an amount of USD 10,000 to USD 100,000 from ZimmerBiomet (Warsaw, IN, USA) and USD 10,000 to USD 100,000 from Link Orthopaedics (Rockaway, NJ, USA). One of the authors (MJT) or a member of his immediate family has or may receive payments or benefits, during the study period, less than USD 10,000 from DJO Orthopedics (Vista, CA, USA). One of the authors (AFK) or a member of his immediate family, has or may receive payments or benefits, during the study period, an amount of less than USD 10,000 from Innomed (Savannah, GA, USA) and has or may receive payments or benefits, during the study period, an amount of less than USD 10,000 from ZimmerBiomet.

All ICMJE Conflict of Interest Forms for authors and Clinical Orthopaedics and Related Research ${ }^{\circledR}$ editors and board members are on file with the publication and can be viewed on request.

Each author certifies that his or her institution approved the human protocol for this investigation, that all investigations were conducted in conformity with ethical principles of research, and that informed consent for participation in the study was obtained.
}

This work was performed at the Mayo Clinic, Rochester, MN, USA.

K. L. Poehling-Monaghan, M. J. Taunton ( $₫)$, R. T. Trousdale, R. J. Sierra, M. W. Pagnano

Mayo Clinic, 200 1st Street SW, Rochester, MN 55905, USA

e-mail: Taunton.michael@mayo.edu
Questions/purposes (1) Do serum markers of inflammation/muscle damage predict pain or early functional outcomes after contemporary THA performed through a direct anterior or miniposterior approach? (2) Do early functional outcomes as measured by in-hospital outcomes and clinical milestones differ between a contemporary direct anterior and miniposterior approach for THA?

Methods Between August 31, 2013, and September 1, 2014, all patients presenting as candidates for THA at our institution who had not already had preoperative blood draws (161) were recruited for this study. Forty-two patients failed these exclusion criteria, eight patients declined enrollment, and 11 were consented but did not complete the required preoperative blood tests. Recruitment stopped when 50 patients had been enrolled in both the direct anterior group and the miniposterior group $(2 \mathrm{n}=$ $100)$ based on a priori power analysis. One high-volume surgeon performed all of the direct anterior approaches and three high-volume surgeons performed the miniposterior approaches. Groups did not differ with the numbers available in mean age (63 years; SD 10; range, 35-86 years), sex (52\% female), or mean body mass index (mean $31 \mathrm{~kg} / \mathrm{m}^{2}$; SD $7 \mathrm{~kg} / \mathrm{m}^{2}$; range, $20-73 \mathrm{~kg} / \mathrm{m}^{2}$ ). Serum markers measured including hemoglobin, hematocrit, myoglobin, creatine kinase (CK), C-reactive protein, interleukin- 6 , and tumor necrosis factor- $\alpha$ were collected at the preoperative clinic visit and on postoperative days 1 and 2 and compared with operative details, in-hospital complications, therapy progress, pain scores, and

\author{
A. F. Kamath \\ Pennsylvania Hospital—Penn Medicine, Philadelphia, PA, USA
}


functional results from a milestone diary. Functional results evaluated included time to discontinue all narcotics and gait aids, independence with activities of daily living, return to driving a motor vehicle, and return to work.

Results Serum markers after contemporary THA were not correlated with early functional outcomes either in-hospital or postdischarge. Specifically, no serum marker was predictive of the time to discontinue gait aids or narcotics, return to driving, climb stairs, or independence in activities of daily living (all $\mathrm{p}>0.08$ ). The patients receiving the direct anterior approach did have lesser elevations of CK levels than the patients undergoing the miniposterior approach $(436 \pm 312$ [direct anterior $\{\mathrm{DA}\}$ ] versus $1071 \pm$ 459 [miniposterior $\{\mathrm{MP}\}$ ], difference in means: -635 ; $95 \%$ confidence interval $[\mathrm{CI}],-809$ to $-462 ; \mathrm{p}<0.001$ ), myoglobin levels $(168 \pm 114[\mathrm{DA}]$ versus $378 \pm 151$ [MP], difference in means: $-210,95 \% \mathrm{CI},-269$ to -151 ; $\mathrm{p}<0.001)$, C-reactive protein $(79 \pm 57$ [DA] versus $124 \pm$ 58 [MP], difference in means: $-46,95 \% \mathrm{CI},-71$ to -21 ; $\mathrm{p}<0.001)$, and interleukin-6 (45 \pm 34 [DA] versus $80 \pm$ 53 [MP], difference in means: $-35,95 \% \mathrm{CI},-54$ to -16 ; $\mathrm{p}<0.001$ ), but not in other serum markers. In the hospital, patients undergoing the direct anterior approach ambulated 35 steps farther with physical therapy (178 feet DA versus 142 feet MP, $\mathrm{p}<0.01$, difference in means: $35,95 \%$ CI, 9$62 ; \mathrm{p}=0.009)$ and had visual analog scale pain scores 1.1 less (4.8 DA versus 5.9 MP, difference in means: -1.1 , 95\% CI, 2.0 to $-0.2 ; \mathrm{p}=0.02$ ) than patients undergoing the miniposterior approach. There were no differences between approaches in other in-hospital outcomes or in posthospital clinical milestones.

Conclusions Serum markers including CK, myoglobin, C-reactive protein, interleukin-6, and tumor necrosis factor- $\alpha$ did not predict early pain/function after contemporary THA approaches. Although lesser elevations in myoglobin, CK, C-reactive protein, and interleukin- 6 were found after direct anterior THA, that difference was not clinically meaningful. Further reporting of serum biomarkers as a measure of physiological burden after orthopaedic surgical procedures should be viewed as suspect until clear linear or threshold values are established.

Level of Evidence Level III, diagnostic study.

\section{Introduction}

The invasiveness of surgery is difficult to measure. The serum enzymes released after damage to skeletal muscle in the setting of cardiac injury, trauma, sports medicine, and rhabdomyolysis have been well documented $[4,7-9,13-$ 16, 18-20]. The specific enzymes released during surgical dissection, however, have been reported only in a select number of studies on the lumbar spine and the hip [14, 15, 18]. Creatine kinase (CK) has been found to rise in a varying pattern, peaking anywhere from 9 to 47 hours after lumbar spine surgery [15]. The validity of its use in measuring surgical damage was suggested when peak serum levels of CK measured 12 to 48 hours after spinal surgery directly correlated with the surface area of muscle dissected and retracted [18]. Also, CK and myoglobin were found to rise after subcostal abdominal approaches and THA with myoglobin peaking on postoperative day 1 [16]. Additionally, serum markers of inflammation and muscle damage have been proposed as a potential measure of muscle damage in both total hip and total knee surgery [3, $4,6,12]$.

Controversy continues over the relative merits of various surgical approaches to THA with proponents of certain methods citing decreased damage to the patient's soft tissues during exposure. One method of quantifying muscle damage has involved MRI evaluation of the soft tissues at 1 year postoperatively with results suggesting that the patients undergoing the direct anterior approach had less soft tissue signal within the abductor musculature [5]. Cadaveric dissection has also been used to visually evaluate the integrity of the soft tissues after varying approaches, quantifying the percentage of specific muscles damaged through each approach. One report noted that less damage occurred in the gluteus minimus muscles and minimus tendon with the direct anterior approach when compared with the posterolateral approach [22]. Another study evaluated serum markers of muscle damage in patients undergoing one of three different approaches to the hip (Watson Jones, miniposterior, and two-incision) and found no difference in trends of serum enzymes [6]. Most recently, however, serum markers of muscle damage and inflammation were reported to be much lower in patients undergoing a direct anterior approach as compared with a posterior approach [3]. Although these various studies have provided a degree of insight into serum inflammatory markers and gross response of the body to surgical trauma, clinical correlation with subsequent functional outcomes for patients is lacking.

No English-language studies of which we are aware explore the relationship between markers of muscle damage after contemporary THA and the subsequent functional outcomes for patients. There may be potential clinical benefit if a threshold level of a serum marker of inflammation or muscle damage after THA could predict postoperative pain or early clinical outcome.

We therefore asked: (1) Do serum markers of inflammation/muscle damage predict pain or early functional outcomes after contemporary THA performed through a direct anterior or miniposterior approach? (2) Do early functional outcomes as measured by a visual analog scale 
(VAS) for pain, distance ambulated in physical therapy, time to discontinue walking aids and narcotics, and various other activities of daily living (ADLs)/hospital measures differ between a contemporary direct anterior and miniposterior approach for THA?

\section{Patients and Methods}

This is a prospective nonrandomized study conducted at an academic tertiary referral center. Between August 31, 2013, and September 1, 2014, we approached all patients presenting as candidates for THA at our institution who had not already had preoperative blood draws (161). Exclusion criteria included patients not undergoing primary THA or those who had already had their routine preoperative blood draws before our discussion for informed consent. A total of 161 patients ages 18 to 100 years were approached for willingness to participate in the study. Forty-two patients failed exclusion criteria (screening failure: patients already had a blood draw), eight patients declined enrollment, and 11 were consented but did not complete the required preoperative blood tests (patients failed to get the blood draw preoperatively). All consenting patients who otherwise met these prespecified criteria were enrolled until the target group size was achieved. Recruitment stopped when 50 patients had been enrolled in both the direct anterior group and the miniposterior group $(2 \mathrm{n}=100)$ based on a priori power analysis. Fifty patients were recruited from the surgeon (MJT) who performs direct anterior and 50 patients were recruited from the surgeons (MWP $\mathrm{n}=22$, RTT $\mathrm{n}=23$, RJS $n=5$ ) who perform miniposterior approaches. All four surgeons are high-volume arthroplasty surgeons who routinely perform the approach in question for primary hip arthroplasty. Patients were excluded if they had a prior procedure on the operative femur or acetabulum, prior trauma or infection to the area, inflammatory arthropathy, surgical intervention within the past 6 weeks, and those who declined enrollment or refused additional blood draws. All THAs were done during the time of the study period. For the purposes of this study, followup was 8 weeks for all patients.

\section{Surgical Technique}

Patients underwent surgery through a miniposterior or direct anterior approach, depending on the preference of their surgeon. During the time of this study, $99 \%$ of primary THAs were performed through a direct anterior approach by MJT and $100 \%$ of primary THAs performed by RTT, RJS, and MWP were performed through a miniposterior approach. The primary THAs that were not performed through a direct anterior approach by MJT were in patients who had previously had surgery on the operative femur, which were already excluded from this study. Before the initiation of the data collection period, all surgeons had performed greater than 200 THAs using their respective technique.

\section{Direct Anterior Approach: Technique}

The patient was positioned in a supine position on an orthopaedic table that allows hyperextension and adduction of the operative extremity. An oblique incision was made over the anterior margin of the tensor muscle at a point approximately $2 \mathrm{~cm}$ lateral from the anterosuperior iliac spine and extending 8 to $10 \mathrm{~cm}$. The fascia of the tensor muscle was identified and incised. The muscle was swept digitally laterally and a retractor was placed over the superior aspect of the femoral neck. The ascending branch of the lateral femoral circumflex artery was identified and cauterized. The hip capsule was then incised and retracted. A measured resection of the femoral neck was performed with the assistance of fluoroscopy and preoperative templates. Acetabular reaming and final component positioning were performed with fluoroscopic assistance and direct visualization. For femoral preparation, the operative extremity was externally rotated, extended, and adducted allowing axial access to the proximal femur. Capsular releases were performed as needed for exposure. The surgical implantation of the femoral implant trial was followed by a trial reduction. The final femoral component sizing, offset, and leg length were evaluated fluoroscopically. After appropriate sizing, the final femoral implants were placed.

\section{Miniposterior Approach: Technique}

The miniposterior surgical approach involved a 7- to 9.5$\mathrm{cm}$ incision along the posterior aspect of the femur, starting at the tip of the greater trochanter and proceeding distally. The fascia of the gluteus maximus was split, and blunt dissection revealed the underlying abductor and external rotator musculature. The external rotators and the hip capsule were incised and preserved as one layer with an attempt being made to preserve the insertion of the quadratus femoris on the femur. The hip was dislocated posteriorly, and the femoral neck was cut in accordance with the preoperative plan. Acetabular retractors were positioned, the acetabulum was reamed, and the real acetabular component was placed. The hip was then flexed, and retractors were placed around the femoral 
neck to allow reaming, broaching, and trial insertion of the femoral component. The femoral component was then impacted into place, the femoral head was assembled, and the hip was reduced. The hip capsule and the external rotators were meticulously repaired back to the greater trochanter through three drill holes with use of nonabsorbable sutures that were placed in a locking-looped fashion.

All patients received the same formal, preoperative class educating them on perioperative expectations and outcomes. All received a preoperative femoral nerveindwelling catheter for 36 hours postoperatively and an oral pain regimen including scheduled acetaminophen with tramadol and narcotics as needed. Patients sat at the edge of the bed the evening of postoperative day 0 and were seen twice daily by therapists beginning on postoperative day 1 . All were allowed to weightbear as tolerated with gait aids and followed the same rapid rehabilitation protocol with no hip precautions enforced.

Markers of hemoglobin, hematocrit, CK, myoglobin, Creactive protein (CRP), tumor necrosis factor- $\alpha$ (TNF- $\alpha$ ), and interleukin-6 (IL-6) (henceforth referred to as "serum markers") were added to routinely scheduled blood draws at the patients' preoperative visit, on the morning of postoperative day 1 , and on the morning of postoperative day 2.

Data collected during their hospital stay included length of stay; number of stairs climbed with physical therapy (PT); maximum number of feet walked per one PT encounter; need for "breakthrough" intravenous opiates; VAS pain score; discharge disposition; and in-hospital complications. Per our protocol, each patient is encouraged to perform stairs, regardless of social situation, as a result of the overall needs of community living. Each patient was encouraged to walk in each PT session. IV opiates were administered on an as-needed basis only and had to be ordered specifically by the surgeon's team. There were no patient-controlled analgesia or scheduled IV narcotics for either group. In-hospital complications recorded included medical complications, wound complications, falls, or any other orthopaedic complication.

Patients were followed daily while in the hospital. Postoperative followups were performed at 8 weeks. At discharge, patients were given a diary to document exactly when they were able to achieve certain milestones that were then queried at their 8-week face-to-face clinic followup. These milestones included time to discontinuation of all narcotics and gait aids, time to independence (ADLs), and time until they could drive a motor vehicle and return to work. The dates recorded in the milestone diary were discussed with the patients and any undocumented information was addressed. Preoperative and 8-week Harris hip scores were also recorded.
Statistical Analysis

The primary outcome was defined as the change in the value of the serum markers (hemoglobin, hematocrit, CK, myoglobin, CRP, TNF- $\alpha$, and IL-6) from baseline (presurgery) to 72 hours postsurgery. Additional outcomes included functional outcomes measured 8 weeks postsurgery. The serum markers were evaluated primarily as continuous variables. The strength of the association between the serum markers and the pain and hip function outcomes was evaluated by calculating correlation coefficients and by performing simple linear regression. In addition, nonlinear regression and graphic examination were also used to examine the relationship between the serum markers and the functional outcomes. The association between the change in marker levels and the type of surgical approach (direct anterior, miniposterior) was evaluated using analysis of variance (ANOVA). Patients in the two surgical approach groups were compared with respect to their demographics (eg, gender, age, body mass index [BMI]) using ANOVA. All statistical tests were twosided and the threshold of statistical significance was set at $\alpha=0.05$.

The primary goal of this study was to evaluate the potential association of the change in biomarker values and functional outcomes after THA. The secondary goal was to compare the patients in the direct anterior group with the patients in the miniposterior group with respect to postoperative serum markers and functional outcomes. Therefore, to address both of these goals, power analyses were performed to calculate sample sizes necessary to have $80 \%$ power to detect meaningful correlations between the biomarker changes and the functional outcomes for the overall cohort as well as to detect between-group differences in functional outcomes. Although what constitutes a meaningful correlation is debatable, we took a conservative approach and calculated the sample size necessary to detect a correlation coefficient of at least 0.3 as being statistically significantly different from 0 . Correlation coefficients range from -1 to +1 with the extreme values of \pm 1 representing a perfect linear relationship (either negative or positive) and a value of 0 suggesting complete lack of a linear relationship. A correlation coefficient of 0.3 is representative of a very weak positive linear relationship. It has been suggested that a correlation less than 0.3 is negligible, whereas correlations of 0.3 to $0.5,0.5$ to $0.7,0.7$ to 0.9 , and 0.9 to 1.0 represent low, moderate, high, and very high linear relationships, respectively [10]. Therefore, by choosing a sample size to have power to detect a correlation of at least 0.3 , we had reasonable power to identify even very weak associations. The power analysis showed that to have $80 \%$ power to detect a correlation coefficient of at least 0.3 as being statistically different from 0 (with $\alpha$ 
Table 1. Preoperative details and Harris hip scores

\begin{tabular}{|c|c|c|c|c|}
\hline Patient details and HHS & Direct anterior $(\mathrm{n}=50)$ & Miniposterior $(\mathrm{n}=50)$ & $\mathrm{p}$ value & All $(n=100)$ \\
\hline \multirow[t]{2}{*}{ Age (years) } & $63(\mathrm{SD} 9.3)$ & $63(\mathrm{SD} 11.3)$ & $0.9825^{\dagger}$ & $63($ SD 10.3) \\
\hline & Range $41-84$ & Range $35-86$ & & Range $35-86$ \\
\hline Gender & $48 \%$ female & $56 \%$ female & $0.4796^{*}$ & $52 \%$ female \\
\hline \multirow[t]{2}{*}{ BMI $\left(\mathrm{kg} / \mathrm{m}^{2}\right)$} & $31(\mathrm{SD} 5.11)$ & $30(\mathrm{SD} 8.09)$ & $0.8584^{\dagger}$ & $31(\mathrm{SD} 6.71)$ \\
\hline & Range $20-44$ & Range $23-73$ & & Range $20-73$ \\
\hline \multirow[t]{2}{*}{ Preoperative HHS } & 75 (SD 5.45) & $70($ SD 7.28) & $0.0002^{\dagger}$ & 73 (SD 6.9) \\
\hline & Range $62-87$ & Range $56-86$ & & Range $56-87$ \\
\hline \multirow[t]{3}{*}{ Postoperative HHS at 8 weeks } & $\mathrm{n}=50$ & $\mathrm{n}=42$ & $0.5602^{\dagger}$ & $\mathrm{n}=92$ \\
\hline & $91(\mathrm{SD} 3.53)$ & $92(\mathrm{SD} 3.43)$ & & $92(\mathrm{SD} 3.5)$ \\
\hline & Range $84-98$ & Range 86-97 & & Range $84-98$ \\
\hline \multirow[t]{3}{*}{ Change in HHS } & $\mathrm{n}=50$ & $\mathrm{n}=41$ & $<0.0001^{\dagger}$ & 19 (SD 6.7) \\
\hline & $16(\mathrm{SD} 5.3)$ & $22(\mathrm{SD} 6.4)$ & & Range 8-34 \\
\hline & Range 8-32 & Range 8-34 & & \\
\hline
\end{tabular}

* Chi-square; ${ }^{\dagger}$ unequal variance t-test; HHS = Harris hip score; BMI = body mass index.

$=0.05$, two-sided test), a sample of 85 patients would be needed. For the secondary goal of comparing functional outcomes between the direct anterior and miniposterior groups, we focused on the number of days to discontinuing gait aids and used estimates from Taunton et al. [24], who reported mean (SD) values of 35.1 (24.6) days for the miniposterior group and 22.8 (11.5) days for the direct anterior group. The difference in these reported means is 12.3 days and the pooled estimate of the SD is 19.2 days. Assuming similar variability were to be observed in this study, a sample of 42 patients in each of the two groups would be needed to have $80 \%$ power to detect a difference between the groups (ie, delta) of at least 12 days in the time to discontinue gait aids ( $\alpha=0.05$, two-sided test). To be conservative and to guard against potential attrition or loss to followup, we increased the sample size of each group to 50 for a total of 100 subjects. Thus, ultimately, with a total of 100 subjects (50 in each group), there was $80 \%$ power to detect correlations of at least 0.3 as being statistically significant and differences of at least 12 days between the direct anterior and miniposterior groups as being statistically significant ( $\alpha=0.05$, two-sided). The patients did not differ in their demographics with a mean age of 63 years (SD 10.3; range, 35-86 years), 52\% female, and an average BMI of $31 \mathrm{~kg} / \mathrm{m}^{2}\left(\mathrm{SD} 6.7 \mathrm{~kg} / \mathrm{m}^{2}\right.$; range, $\left.19-73 \mathrm{~kg} / \mathrm{m}^{2}\right)$. The two groups are compared (Table 1).

A total of 100 patients were enrolled in the study and completed 8 weeks of followup. No patients were lost to followup. Not all functional outcomes were available for all patients. Although 8-week followup was obtained, some patients did not report their entire diary (Table 2), and some patients did not have an 8-week Harris hip score calculated (Table 1). Study followup was terminated at the 8 -week recheck. There was no intention-to-treat or per- protocol analysis performed, because the study design did not allow crossover.

\section{Results}

There was no correlation with postoperative serum marker levels (hemoglobin, hematocrit, CK, myoglobin, CRP, TNF- $\alpha$, and IL-6) and binary or continuous outcomes for both in-hospital outcomes and clinical milestones, regardless of approach. Correlations were determined by calculating odds ratios for association of a set threshold change in serum biomarkers (CK $500 \mathrm{IU} / \mathrm{L}, \mathrm{CRP} 100 \mathrm{mg} /$ $\mathrm{dL}$, hematocrit $8 \%$, hemoglobin $2 \mathrm{~g} / \mathrm{dL}$, IL-6 $50 \mathrm{ng} / \mathrm{L}$, myoglobin $250 \mathrm{ng} / \mathrm{mL}$, and TNF- $\alpha 0.25 \mathrm{pg} / \mathrm{mL}$ ) with binary outcomes (Table 3). Additionally, the sample correlation with the changes in serum biomarkers with continuous outcomes were calculated (Table 4). There was no correlation between functional outcomes and serum marker levels (Figs. 1, 2).

Patients who received the miniposterior approach had a greater rise in CK after surgery than did patients treated with the direct anterior approach $(436 \pm 312$ versus $1071 \pm 459$, difference in means: $-635,95 \%$ confidence interval $[\mathrm{CI}],-809$ to $-462 ; \mathrm{p}<0.001)$; similar greater rises were observed for myoglobin $(168 \pm 114$ versus 378 \pm 151 , difference in means: $-210,95 \% \mathrm{CI},-269$ to -151 ; $\mathrm{p}<0.001)$, CRP $(79 \pm 57$ versus $124 \pm 58$, difference in means: $-46,95 \% \mathrm{CI},-71$ to $-21 ; \mathrm{p}<0.001$ ), and for IL-6 ( $45 \pm 34$ versus $80 \pm 53$, difference in means: $-35,95 \%$ $\mathrm{CI},-54$ to $-16 ; \mathrm{p}<0.001)$. However, there was no difference in the decrease of hemoglobin $(3.0 \pm 0.8$ versus 2.6 \pm 1.6 , difference in means: $0.3,95 \% \mathrm{CI},-0.2$ to $0.8 ; \mathrm{p}=$ $0.177)$ or hematocrit $(8.6 \pm 2.8$ versus $7.9 \pm 4.0$, difference 
Table 2. Functional outcomes by approach

\begin{tabular}{|c|c|c|c|c|}
\hline Mean days (SD) & Direct anterior $(\mathrm{n}=50)$ & Posterior $(\mathrm{n}=50)$ & Difference in means, $95 \% \mathrm{CI}$ & All \\
\hline \multirow[t]{3}{*}{ Using walker } & $\mathrm{n}=46$ & $\mathrm{n}=43$ & $-1.028(-5.744$ to 3.689$) ; \mathrm{p}=0.6658^{*}$ & $\mathrm{n}=89$ \\
\hline & $15(10.3)$ & $16(12.0)$ & & $16(11.1)$ \\
\hline & Range $2-49$ & Range $2-51$ & & Range $2-51$ \\
\hline \multirow[t]{3}{*}{ Using walking aids } & $\mathrm{n}=46$ & $\mathrm{n}=42$ & $-0.850(-8.471$ to 6.771$) ; \mathrm{p}=0.8250^{*}$ & $\mathrm{n}=88$ \\
\hline & $27(17.4)$ & $28(18.4)$ & & $27(17.8)$ \\
\hline & Range 3-84 & Range 6-100 & & Range 3-100 \\
\hline \multirow[t]{3}{*}{ Using narcotics } & $\mathrm{n}=46$ & $\mathrm{n}=44$ & $-1.427(-6.727$ to 3.873$) ; p=0.5939^{*}$ & $\mathrm{n}=90$ \\
\hline & $10(12.3)$ & $12(13.0)$ & & $11(12.6)$ \\
\hline & Range $0-63$ & Range $0-60$ & & Range $0-63$ \\
\hline \multirow[t]{3}{*}{ To resume ADLs } & $\mathrm{n}=46$ & $\mathrm{n}=44$ & $2.477(-2.715$ to 7.669$) ; p=0.6507^{\dagger}$ & $\mathrm{n}=90$ \\
\hline & $15(14.8)$ & $12(9.5)$ & & $13(12.5)$ \\
\hline & Range $0-80$ & Range $1-42$ & & Range $0-80$ \\
\hline \multirow[t]{3}{*}{ To climb stairs } & $\mathrm{n}=45$ & $\mathrm{n}=42$ & $4.389(-2.920$ to 11.698$) ; \mathrm{p}=0.4287^{\dagger}$ & $\mathrm{n}=87$ \\
\hline & $15(17.9)$ & $10(16.0)$ & & $12(17.1)$ \\
\hline & Range $0-87$ & Range 1-94 & & Range 0-94 \\
\hline \multirow[t]{3}{*}{ To drive car } & $\mathrm{n}=46$ & $\mathrm{n}=44$ & $1.262(-4.616$ to 7.140$) ; \mathrm{p}=0.6707^{*}$ & $\mathrm{n}=90$ \\
\hline & $22(14.1)$ & $21(14)$ & & $22(14)$ \\
\hline & Range 2-60 & Range 1-94 & & Range 2-60 \\
\hline \multirow[t]{3}{*}{ To return to work } & $\mathrm{n}=30$ & $\mathrm{n}=27$ & $11.870(0.845-22.895) ; \mathrm{p}=0.0354^{*}$ & $\mathrm{n}=57$ \\
\hline & $38(24.8)$ & $26(16.1)$ & & $33(21.8)$ \\
\hline & Range $3-100$ & Range 3-60 & & Range $3-100$ \\
\hline \multirow[t]{3}{*}{ To walk 0.5 mile } & $\mathrm{n}=44$ & $\mathrm{n}=30$ & $5.718(-2.408$ to 13.844$) ; \mathrm{p}=0.1650^{*}$ & $\mathrm{n}=73$ \\
\hline & $30(22.4)$ & $24(14.3)$ & & $27(19.1)$ \\
\hline & Range 3-90 & Range $7-56$ & & Range 3-90 \\
\hline
\end{tabular}

* Unequal variance t-test; ${ }^{\dagger}$ Wilcoxon; $\mathrm{CI}=$ confidence interval; ADLs = activities of daily living.

in means: $0.7,95 \% \mathrm{CI},-0.7$ to $2.0 ; \mathrm{p}=0.345)$ or in the increase of TNF- $\alpha(0.4 \pm 0.9$ versus $0.5 \pm 0.4$, mean difference with $95 \% \mathrm{CI} ; \mathrm{p}=0.8948$ ) (Table 5).

Patients receiving the direct anterior approach ambulated further in the hospital with PT than patients receiving the miniposterior approach $(178 \pm 74$ feet versus $142 \pm 58$ feet, difference in means: $35,95 \% \mathrm{CI}, 9$ to $-62 ; \mathrm{p}=0.01$ ) and had lower VAS pain in the hospital $(4.8 \pm 2.6$ versus $5.9 \pm 2.1$, difference in means: $-1.1,95 \% \mathrm{CI},-2.0$ to $-0.2, \mathrm{p}=0.02$ ), although below the minimal clinically important difference (MCID) [17]. There were two (4\%) patients undergoing the miniposterior approach who had in-hospital complications of atrial fibrillation versus no complications in the direct anterior group $(p=0.02)$. However, there were no differences noted in the other hospital outcomes (Table 6) or in the time to reach clinical milestones (Table 2).

\section{Discussion}

There is continued debate over the purported benefits of less invasive approaches for hip arthroplasty. Some reports
[1, 3, 11] have focused attention on serum markers of muscle damage, and there has been some suggestion that lesser elevation in selected serum markers in musclesparing approaches is indicative of less overall damage to the patient and may be associated with reduced recovery time. Specifically, Bergin et al. [3] demonstrated the average increase in CK level in posterior approaches was 5.5 times higher than anterior approaches with a mean difference of 150.3 units/L (95\% CI, 70.4-230.2). The difference in the cumulative rise of $\mathrm{CK}$ also reached significance, nearly twice as high in the posterior group (mean difference, 305.0 units/L; $95 \% \mathrm{CI},-46.7$ to 656.8 ). There was no correlation with the differences in serum markers and hospital outcomes or clinical milestones, but a rise in $\mathrm{CK}$ was found to be independently associated with surgical approach. We know of no other English-language studies that explore the relationship between markers of muscle damage after varying approaches to the hip and actual patient outcomes. We believed there might be clinical benefit if a threshold level of a serum marker of inflammation or muscle damage after THA could help predict pain or clinical outcome. However, this study demonstrated no correlation between postoperative changes in common 


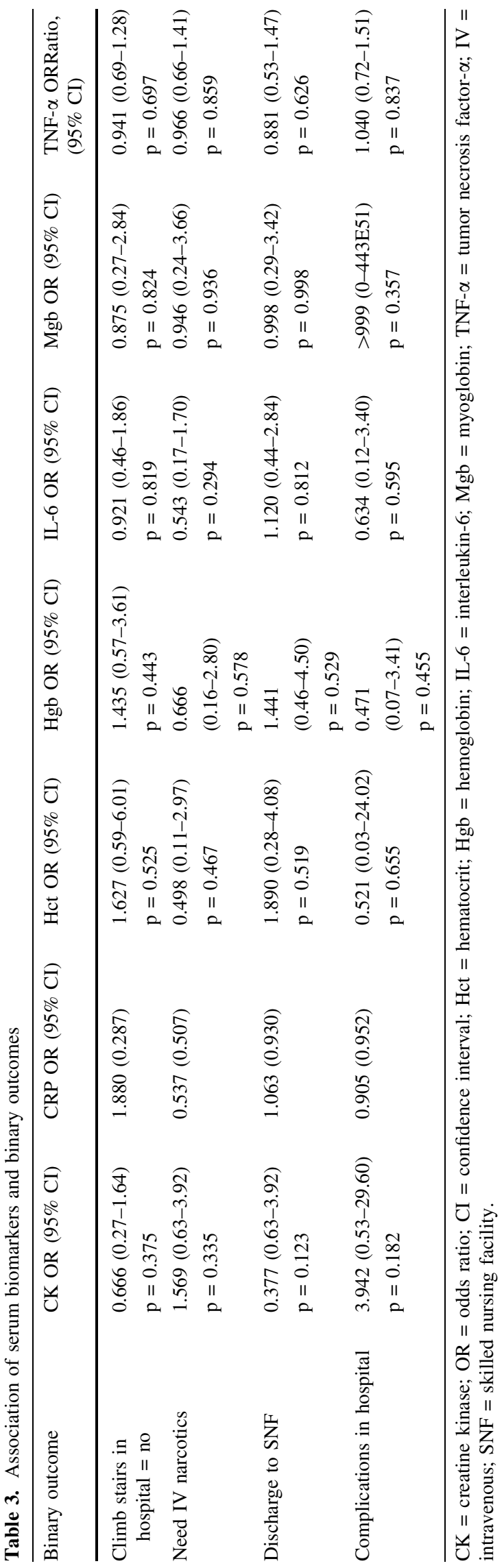

serum markers and clinical outcomes in the early postoperative period after two common less invasive approaches to THA.

This study had a number of limitations. First, this is not a randomized cohort of patients, which could introduce bias into the study that has not been noted in the statistical analysis. However, the cohorts are well matched in age, gender, and BMI (Table 1). These were also not a selected group of patients, but a consecutive cohort of patients who met the study criteria. Second, the procedures were performed by different surgeons, which could add to the clinical outcome variability in the patient cohorts. One surgeon, who was well outside his learning curve, performed all of the direct anterior procedures. Three senior surgeons who all used a very similar surgical technique performed the miniposterior approach procedures. The physical therapy protocols for both cohorts were identical and performed by the same physical therapists with no variation noted for approach. Social workers applied the same discharge criteria for all patients, and a majority ( $92 \%$ of each group) was discharged to home in both cohorts. Third, the biomarkers measured may be the incorrect markers to be measured to predict clinical outcome. If there is a biomarker that we have not identified or tested, that could imply that the findings of the article are incorrect. However, we tested widely accepted, relevant biomarkers of muscle damage and inflammation. Fourth, there is a potential of recall bias at the 8-week followup. To combat that, we did have the patients fill out a diary to document their clinical milestones as they were achieved. There is no reason to suspect there would be any variation in reporting among the groups. Fifth, the in-hospital and functional endpoints studied may be too crude to quantify the level of "invasiveness," which is what these serum markers seek to quantify, and so this could account for the lack of correlation. However, these functional endpoints appear valid and have been accepted as clinically important in prior studies.

This study was powered to find a correlation between serum markers and clinical outcome and secondarily a difference between the direct anterior and miniposterior approach with regard to biomarker levels and postoperative function. This study was not designed to nor is it able to comment on safety. It may also be subject to beta error for outcomes such as infection resulting from the small sample size. Although serum markers of inflammation and muscle damage have been proposed as objective evidence of the soft tissue damage at the time of THA [3], we could find no evidence of a correlation between serum marker elevation and early functional outcomes (Tables 3, 4; Figs. 1,2). We also could find no English-language studies that have prospectively studied the relationship of serum markers and clinical outcomes. The implications of muscle damage, 


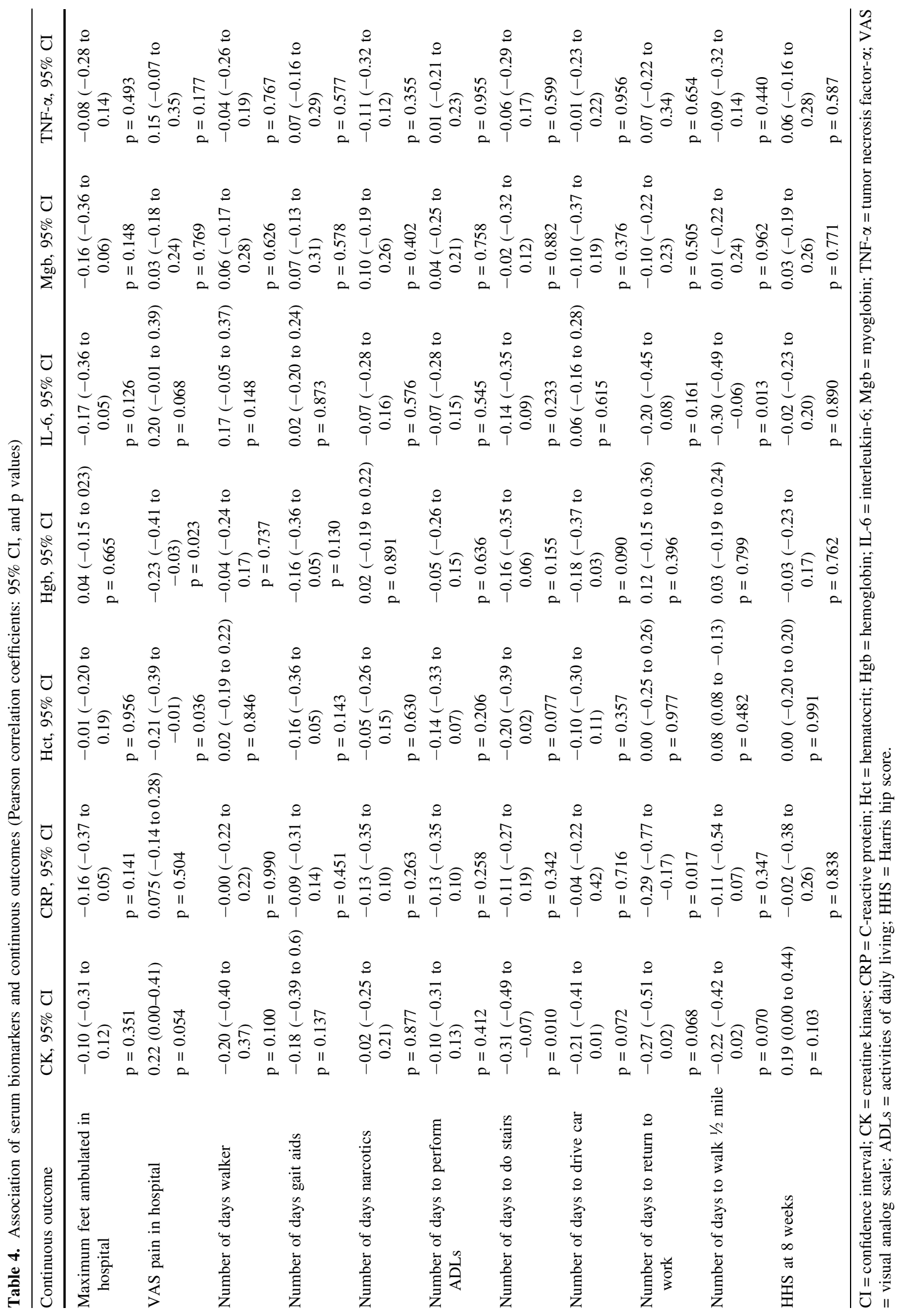


Fig. 1 The correlation of days using walking aids and change in $\mathrm{CK}$ is shown.

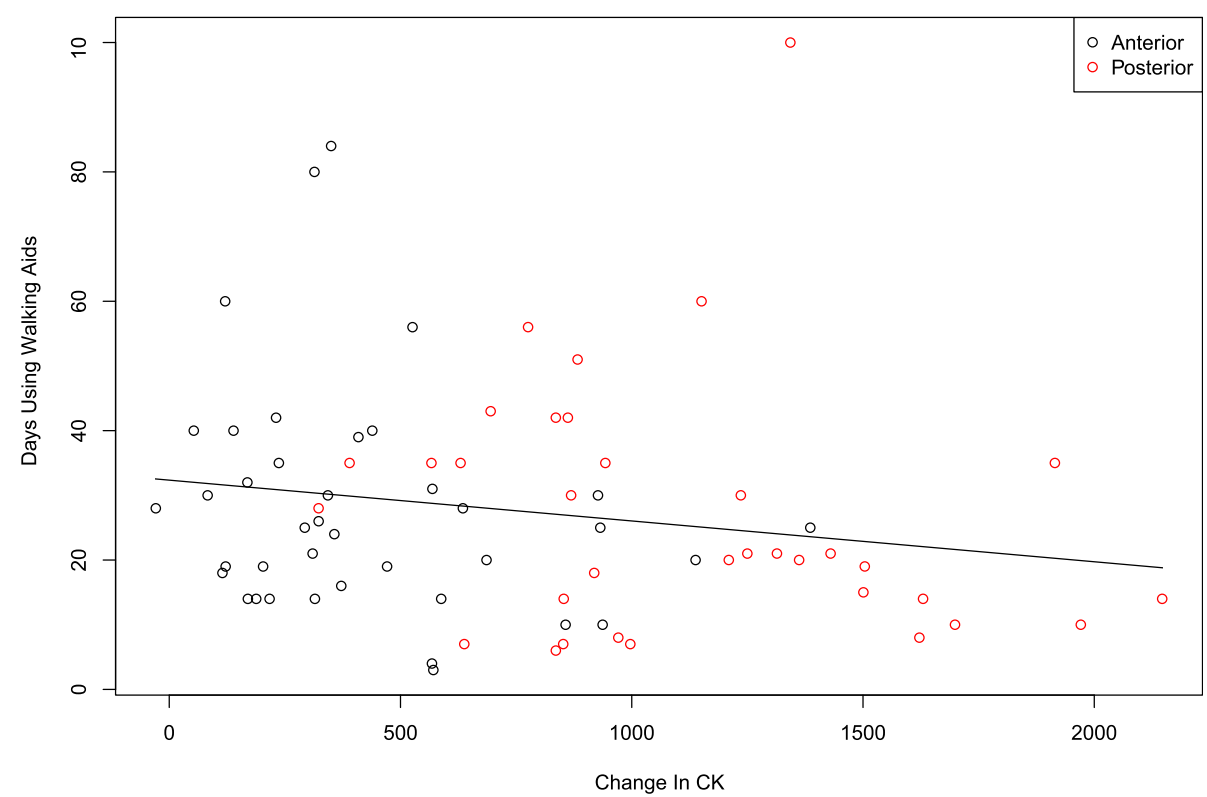

Fig. 2 The correlation of days using walking aids and change in myoglobin is shown.

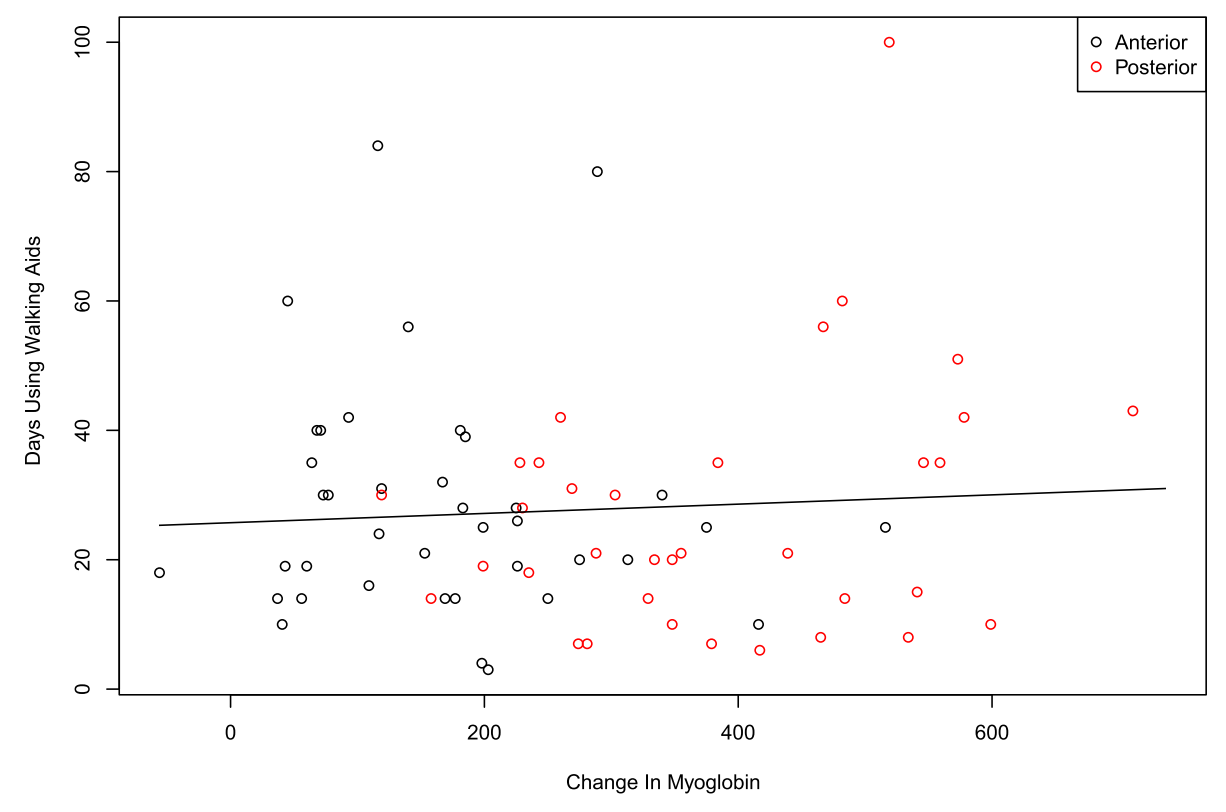

as measured by such serum markers, during THA have yet to be fully quantified. Additionally, there is no known linear relationship that exists between the amount of serum marker elevation and the severity of muscle fiber insult. This suggests that perhaps increases in these markers are indicative of a more extensive muscle manipulation but not necessarily a clinically important insult. In the neurosurgical literature, $\mathrm{CK}$ measured during intracranial cases in the lateral position were found to be $1763 \mathrm{UI} / \mathrm{L}( \pm 1905)$ on postoperative day 2 for procedures that averaged 5.4 hours in duration. Factors dictating a rise in CK were positively correlated with duration of surgery and BMI and negatively with age [23]. This is compared with the results of the present study with an average CK of $1071 \mathrm{UI} / \mathrm{L}$ for patients undergoing the miniposterior approach operated in the lateral decubitus position and $436 \mathrm{UI} / \mathrm{L}$ for the patients undergoing the direct anterior approach in the supine position postoperatively. Because $\mathrm{CK}$ is frequently used as a sensitive biomarker of muscle injury, this comparison notes that the overall $\mathrm{CK}$ release, and perhaps muscle injury, in THA in the lateral position is less than that for a patient in the lateral position for 5 hours alone for a neurosurgical procedure, where no muscle is manipulated. Were this damage clinically important, one would expect patients to have more early postoperative pain or to mobilize more slowly.

When the cohort of 100 patients is separated into the two groups of miniposterior and direct anterior approaches 
Table 5. Serum biomarker changes by approach

\begin{tabular}{|c|c|c|c|c|c|c|c|}
\hline $\begin{array}{l}\text { Biomarkers: } \\
\text { mean }(\mathrm{SD})\end{array}$ & $\mathrm{CK}$ & Myoglobin & CRP & IL-6 & $\mathrm{Hgb}$ & Hct & TNF- $\alpha$ \\
\hline Direct anterior & $436(312)$ & 168 (114) & $79(57.0)$ & 45 (34.3) & $3(0.8)$ & $9(2.8)$ & $0.4(0.9)$ \\
\hline Miniposterior & $1071(459)$ & $378(151)$ & $124(57.6)$ & $80(53.2)$ & $3(1.6)$ & $8(4.0)$ & $0.5(0.4)$ \\
\hline $\begin{array}{l}\text { Difference in means: } \\
\text { DA versus MP }(95 \% \mathrm{CI})\end{array}$ & $\begin{array}{l}-635(-809.1 \\
\quad \text { to }-461.5) \\
\mathrm{p}<0.001\end{array}$ & $\begin{array}{r}-210(-268.6 \\
\quad \text { to }-151.3) ; \\
p<0.001\end{array}$ & $\begin{array}{l}-46(-70.7 \\
\quad \text { to }-20.7) \\
p<0.001\end{array}$ & $\begin{array}{l}-35(-54.0 \\
\quad \text { to }-15.9) \\
\mathrm{p}<0.001\end{array}$ & $\begin{array}{l}0.3(-0.2 \text { to } 0.8) \\
p=0.177\end{array}$ & $\begin{array}{l}0.7(-0.7 \text { to } 2.0) \\
p=0.345\end{array}$ & $\begin{array}{l}-0.02(-0.3 \text { to } 0.3) ; \\
p=0.895\end{array}$ \\
\hline
\end{tabular}

$\mathrm{CK}=$ creatine kinase; $\mathrm{CRP}=\mathrm{C}$-reactive protein; IL-6 = interleukin-6; Hgb = hemoglobin; Hct = hematocrit; TNF- $\alpha=$ tumor necrosis factor- $\alpha$; $\mathrm{DA}=$ direct anterior; $\mathrm{MP}=$ miniposterior .

Table 6. In-hospital outcomes by approach

\begin{tabular}{|c|c|c|c|c|}
\hline In-hospital outcome & Direct anterior $(\mathrm{n}=50)$ & Posterior $(n=50)$ & $\begin{array}{l}\text { Difference in means or odds ratio, } \\
95 \% \mathrm{CI} ; \mathrm{p} \text { value }\end{array}$ & All \\
\hline Climb stairs & $45(90 \%)$ & $42(84 \%)$ & $\begin{array}{l}\text { OR: } 1.50,95 \% \mathrm{CI}-0.44 \text { to } 5.09 \\
\mathrm{p}=0.514 *\end{array}$ & $87(87 \%)$ \\
\hline $\begin{array}{l}\text { Maximum feet ambulated } \\
\text { with PT, mean (SD) }\end{array}$ & $\begin{array}{l}178(74.2) \\
\text { Range } 85-450\end{array}$ & 142 (57.7) Range 50-250 & $\begin{array}{l}\text { Difference in means: } 35.396 \\
\quad 95 \% \text { CI 8.906-61.886; } \\
\mathrm{p}=0.009^{\dagger}\end{array}$ & $\begin{array}{l}160(\text { SD } 68.5) \\
\text { Range } 50-450\end{array}$ \\
\hline IV narcotics needed, number $(\%)$ & $2(4 \%)$ & $3(6 \%)$ & $\begin{array}{l}\text { OR: } 1.57,95 \% \text { CI } 0.25-9.80 \\
p=0.678^{\dagger}\end{array}$ & $5(5 \%)$ \\
\hline VAS pain score (SD) & $\begin{array}{l}5(2.6) \\
\text { Range } 1-9\end{array}$ & $\begin{array}{l}6(2.1) \\
\text { Range } 1-9\end{array}$ & $\begin{array}{l}\text { Difference in means: }-1.098,95 \% \\
\quad \mathrm{CI}-2.029 \text { to }-0.166 ; \\
\mathrm{p}=0.021^{\dagger}\end{array}$ & $\begin{array}{l}5(2.3) \\
\text { Range } 1-9\end{array}$ \\
\hline $\begin{array}{l}\text { Number of complications } \\
\text { in hospital }\end{array}$ & $0(0 \%)$ & $2(4 \%)$ & $\begin{array}{l}\text { OR: } 5.43,95 \% \text { CI } 0.25-119.71 ; \\
p=0.021^{*}\end{array}$ & $2(2 \%)$ \\
\hline Length of stay (days) & $\begin{array}{l}1.92(\text { SD } 0.67) \\
\text { Range } 1-4\end{array}$ & $\begin{array}{l}2.08 \text { SD }(0.44) \\
\text { Range } 1-3\end{array}$ & $\begin{array}{l}\text { Difference in means: }-0.202,95 \% \\
\quad \mathrm{CI}-0.454 \text { to } 0.051 ; \\
\mathrm{p}=0.080^{\dagger}\end{array}$ & $\begin{array}{l}1.99 \text { (SD 0.57) } \\
\text { Range } 1-4\end{array}$ \\
\hline Discharge disposition to home & $46(92 \%)$ & $46(92 \%)$ & $\begin{array}{l}\text { OR: } 1.33,95 \% \text { CI } 0.28-6.29 \\
p>0.999^{\ddagger}\end{array}$ & $92(92 \%)$ \\
\hline
\end{tabular}

* Chi-square; ${ }^{\dagger}$ unequal variance t-test; ${ }^{\dagger}$ Fisher's exact; $\mathrm{CI}=$ confidence interval; $\mathrm{PT}=$ physical therapy; IV = intravenous; VAS = visual analog scale; $\mathrm{OR}=$ odds ratio.

for THA, the results are surprising. Despite a smaller CK and myoglobin changes in the direct anterior approach group, the only clinical difference was an increase in walking in-hospital with PT of an average of 35 feet and a decrease in average VAS pain score in-hospital of 1 point (Table 6). The clinical relevance of these findings is unclear because we are unaware of a MCID for early ambulation and because the difference in VAS pain is below the MCID for that instrument [17]. This is contrasted with Mayr et al. [21] who conducted a prospective randomized study comparing the minimally invasive anterior approach with an anterolateral approach and found that patients undergoing the direct anterior approach improved over a larger number of gait parameters than patients receiving the traditional anterolateral approach. This may indicate that the disruption of the abductors in the anterolateral approach may have more of an effect on postoperative function than the miniposterior approach. Barrett et al. [2] conducted a randomized prospective study of 43 direct anterior and 44 posterolateral THAs using standardized pain and rapid rehabilitation protocols and found lower pain scores in-hospital and more stairs climbed in the direct anterior groups up to 3 months postoperatively. Again, the VAS score differences were less than the MCID for that instrument [17]. However, we were surprised to find that a similar comparison implementing a standardized advanced pain regimen and rapid rehabilitation protocol would offer little differences in the early postoperative period.

In conclusion, the observed changes in serum markers measured had no demonstrable impact on clinical outcomes. The impact of muscle damage on patient outcomes 
in contemporary hip arthroplasty may be less important than previously thought. Surgeons should consider pain and rehabilitation protocols, patient education, and a consistently applied surgical technique when determining which approach may yield the best outcome. Serum markers of muscle damage/inflammation as a measure of physiological burden after orthopaedic surgical procedures appear to have little value until clear linear or threshold values that predict a clinically meaningful functional difference are established.

Acknowledgments We thank Dirk Larson MS, for assistance with statistical assessment and review.

\section{References}

1. Bamman MM, Ferrando AA, Evans RP, Stec MJ, Kelly NA, Gruenwald JM, Corrick KL, Trump JR, Singh JA. Muscle inflammation susceptibility: a prognostic index of recovery potential after hip arthroplasty? Am J Physiol Endocrinol Metab. 2015;308:E670-679.

2. Barrett WP, Turner SE, Leopold JP. Prospective randomized study of direct anterior vs postero-lateral approach for total hip arthroplasty. J Arthroplasty. 2013;28:1634-1638.

3. Bergin PF, Doppelt JD, Kephart CJ, Benke MT, Graeter JH, Holmes AS, Haleem-Smith H, Tuan RS, Unger AS. Comparison of minimally invasive direct anterior versus posterior total hip arthroplasty based on inflammation and muscle damage markers. J Bone Joint Surg Am. 2011;93:1392-1398.

4. Brancaccio P, Lippi G, Maffulli N. Biochemical markers of muscular damage. Clin Chem Lab Med. 2010;48:757-767.

5. Bremer AK, Kalberer F, Pfirrmann CW, Dora C. Soft-tissue changes in hip abductor muscles and tendons after total hip replacement: comparison between the direct anterior and the transgluteal approaches. J Bone Joint Surg Br. 2011;93:886-889.

6. Cohen RG, Katz JA, Skrepnik NV. The relationship between skeletal muscle serum markers and primary THA: a pilot study. Clin Orthop Relat Res. 2009;467:1747-1752.

7. de Meijer AR, Fikkers BG, de Keijzer MH, van Engelen BG, Drenth JP. Serum creatine kinase as predictor of clinical course in rhabdomyolysis: a 5-year intensive care survey. Intensive Care Med. 2003;29:1121-1125.

8. Grande M, Tucci GF, Adorisio O, Barini A, Rulli F, Neri A, Franchi F, Farinon AM. Systemic acute-phase response after laparoscopic and open cholecystectomy. Surg Endosc. 2002;16:313-316.

9. Healey JH, Kagen LJ, Velis KP, Levine DB. Creatine kinase MB in skeletal muscle and serum of spine-fusion patients. Clin Orthop Relat Res. 1985;195:282-288.
10. Hinkle DE, Wiersma W, Jurs SG. Applied Statistics for the Behavioral Sciences. Boston, MA, USA: Houghton Mifflin; 2003.

11. Holm B, Thorborg K, Husted H, Kehlet H, Bandholm T. Surgeryinduced changes and early recovery of hip-muscle strength, legpress power, and functional performance after fast-track total hip arthroplasty: a prospective cohort study. PLoS One. 2013;8:e62109.

12. Huang Z, Shen B, Ma J, Yang J, Zhou Z, Kang P, Pei F. Minimidvastus versus medial parapatellar approach in TKA: muscle damage and inflammation markers. Orthopedics. 2012;35:e10381045.

13. Kodatsch I, Finsterer J, Stollberger C. Serum creatine kinase elevation in a medical department. Acta Med Aust. 2001;28:1115.

14. Kumbhare D, Parkinson W, Dunlop B. Validity of serum creatine kinase as a measure of muscle injury produced by lumbar surgery. J Spinal Disord Tech. 2008;21:49-54.

15. Kumbhare D, Parkinson W, Dunlop B, Ryan E, Denkers M, Shah AA, Bobba R, Adachi J. Biochemical measurement of muscle injury created by lumbar surgery. Clin Invest Med. 2007;30:1220.

16. Laurence AS. Serum myoglobin and creatine kinase following surgery. Br J Anaesth. 2000;84:763-766.

17. Lee JS, Hobden E, Stiell IG, Wells GA. Clinically important change in the visual analog scale after adequate pain control. Acad Emerg Med. 2003;10:1128-1130.

18. Lenke LG, Bridwell KH, Jaffe AS. Increase in creatine kinase MB isoenzyme levels after spinal surgery. J Spinal Disord. 1994;7:70-76.

19. Malik E, Buchweitz O, Muller-Steinhardt M, Kressin P, Meyhofer-Malik A, Diedrich K. Prospective evaluation of the systemic immune response following abdominal, vaginal, and laparoscopically assisted vaginal hysterectomy. Surg Endosc. 2001;15:463-466.

20. Malinoski DJ, Slater MS, Mullins RJ. Crush injury and rhabdomyolysis. Crit Care Clin. 2004;20:171-192.

21. Mayr E, Nogler M, Benedetti MG, Kessler O, Reinthaler A, Krismer M, Leardini A. A prospective randomized assessment of earlier functional recovery in THA patients treated by minimally invasive direct anterior approach: a gait analysis study. Clin Biomech (Bristol, Avon). 2009;24:812-818.

22. Meneghini RM, Pagnano MW, Trousdale RT, Hozack WJ. Muscle damage during MIS total hip arthroplasty: Smith-Petersen versus posterior approach. Clin Orthop Relat Res. 2006;453:293298.

23. Neidert MC, Losa M, Regli L, Sarnthein J. Elevated serum creatine kinase after neurosurgeries in lateral position with intraoperative neurophysiological monitoring is associated with OP duration, BMI and age. Clin Neurophysiol. 2015;126:20262032.

24. Taunton MJ, Mason JB, Odum SM, Springer BD. Direct anterior total hip arthroplasty yields more rapid voluntary cessation of all walking aids: a prospective, randomized clinical trial. J Arthroplasty. 2014;29:169-172. 